Bio - grafía. Escritos sobre la Biología y su Enseñanza. ISSN 2027-1034

Edición Extraordinaria. p.p. 1469 - 1476

Memorias del IX Encuentro Nacional de Experiencias en Enseñanza de la Biología y la Educación Ambiental. IV Congreso Nacional de Investigación en Enseñanza de la Biología.

\title{
RELACIÓN ESCUELA - ZOOLÓGICO: POSIBILIDADES Y DESAFÍOS EN LA PRÁCTICA Y EN LA INVESTIGACIÓN
}

\section{SCHOOL - ZOOS RELATIONSHIP: POSSIBILTIES AND CHALLENGES IN PRACTICE AND RESEARCH}

\author{
Catalina Rodríguez Álvarez ${ }^{1}$ \\ Paola Gómez Ovalle ${ }^{2}$ \\ Yonier Alexander Orozco Marín ${ }^{3}$
}

\section{RESUMEN}

La enseñanza de la Biología y la Educación Ambiental son áreas consideradas de gran importancia para la formación de ciudadanos críticos, que además de la comprensión de diversos fenómenos de la Biología y de corte ambiental, también consigan posicionarse y formular soluciones a los problemas ambientales, aspecto que sugiere la necesidad de innovación en las prácticas educativas en la educación formal. En las últimas décadas viene siendo reconocido el papel fundamental que los espacios no formales de educación pueden desempeñar en la formación de ciudadanos más conscientes de problemas ambientales de su contexto y el conocimiento de alternativas para el cuidado del medio en el que vivimos. En un país biodiverso como Colombia, los Zoológicos cumplen un papel fundamental en trabajos de investigación sobre la biodiversidad y la educación de poblaciones de los grandes centros urbanos. Sin embargo, la investigación desde al área de la didáctica sobre los procesos de enseñanza y aprendizaje en estos espacios aun es escasa en el país. Esta propuesta de taller pretende discutir la importancia de los Zoológicos como espacios no formales de educación, las posibilidades y desafíos de su relación con la escuela y motivar a profesores e investigadores del área a interesarse por este campo de investigación.

PALABRAS CLAVE: Educación en Zoológicos, Espacios de educación no formal, Relación Escuela zoológicos.

\section{ABSTRACT}

The teaching of biology and environmental education are areas considered of great importance for the formation of critical citizens, who, in addition to the understanding of various phenomena of biology and environmental cutting, are also able to position themselves and formulate solutions to environmental problems, which suggests the need for innovation in educational practices in formal education. In recent decades, the

\footnotetext{
${ }^{1}$ Departamento de Educación y Conservación Parque Jaime Duque, Bioparque Wacatá. crodriguez@parquejaimeduque.com

2 Departamento Educación y Conservación Parque Jaime Duque, Bioparque Wacatá. pgomez@parquejaimeduque.com

${ }^{3}$ Mestrado Profissional Em Ensino de Ciências e Matemáticas- Universidade Federal do Acre apmusicomano@gmail.com
} 
Bio - grafía. Escritos sobre la Biología y su Enseñanza. ISSN 2027-1034

Edición Extraordinaria. p.p. $1469-1476$

Memorias del IX Encuentro Nacional de Experiencias en Enseñanza de la Biología y la Educación Ambiental. IV Congreso Nacional de Investigación en Enseñanza de la Biología.

fundamental role that non-formal education spaces can play in the formation of citizens who are more aware of environmental problems in their context and knowledge of alternatives for the care of the environment in which we live has been recognized. In a biodiverse country like Colombia, zoos play a fundamental role in research on biodiversity and education of populations of large urban centers, however, research from the area of didactics on teaching and learning processes in these spaces are still scarce in the country. This workshop proposal aims to discuss the importance of zoos as non-formal educational spaces, the possibilities and challenges of their relationship with the school and motivate teachers and researchers in the area to be interested in this field of research.

KEY-WORDS: Education in Zoos, Non-Formal Education Spaces.

\section{INTRODUCCIÓN}

La enseñanza de la biología y la educación ambiental son áreas de investigación de la enseñanza de las ciencias relativamente recientes. Con el avance de la investigación se ha reconocido la importancia de la educación en estos campos para la formación de ciudadanos críticos y responsables con el medio ambiente. Las cuestiones ambientales relacionadas con la tecnología de hoy en día requieren de la escuela un papel diferente y acciones innovadoras para la formación y la inmersión de los niños y los jóvenes en la cultura científica. La preocupación ya no es exclusivamente la mera transmisión de contenido biológico y ambiental, sino que se busca avanzar hacia la formación del pensamiento crítico, la capacidad de razonamiento y la investigación, así como la promoción de actitudes que permiten una mejor convivencia entre las personas y entre las personas con el medio ambiente.

La preocupación por articular el contenido biológico con las realidades sociales y ambientales de los contextos escolares nos permite entender que la enseñanza y el aprendizaje no ocurren únicamente en la escuela. Las experiencias de la vida cotidiana de los estudiantes y la relación con otros espacios educativos son esenciales para lograr estos objetivos. La necesidad de esta relación parece ser aún más justificada cuando Cazelli (2005) señala que la investigación muestra que por lo general sólo a través de la escuela, los niños y jóvenes en desventaja económica consiguen visitar instituciones culturales (Museos, Zoológicos, Jardines botánicos).

Existen varias formas de educación clasificadas en la literatura como: educación formal, no formal e informal. Bianconi y Caruso (2005) proponen que la educación formal se puede resumir como una que está presente en la educación institucionalizada, gradual cronológicamente y jerárquicamente estructurada y la informal como aquella en la que cada persona adquiere y acumula conocimiento a través de la experiencia cotidiana en el hogar, en el trabajo y en el ocio. La educación no formal, sin embargo, se define como cualquier intento educativo sistemático y 
Bio - grafía. Escritos sobre la Biología y su Enseñanza. ISSN 2027-1034

Edición Extraordinaria. p.p. 1469 - 1476

Memorias del IX Encuentro Nacional de Experiencias en Enseñanza de la Biología y la Educación Ambiental. IV Congreso Nacional de Investigación en Enseñanza de la Biología.

organizado que normalmente se lleva a cabo fuera del marco del sistema de educación formal.

\section{RELACIÓN DE CONTINUIDAD ENTRE LAEDUCACIÓN FORMAL Y NO FORMAL}

Los espacios no formales de educación pueden ser agrupados en institucionalizados y no institucionalizados (Fachín et al, 2014). Los espacios institucionalizados generalmente disponen de infraestructura física, planeamiento y monitores especializados para la labor de mediación, por ejemplo, los Museos, los Zoológicos, los Acuarios, los Centros de ciencia. Plazas públicas, ríos, quebradas, parques y áreas naturales pueden ser consideradas espacios no formales de educación no institucionalizados.

La relación entre la educación desarrollada en contextos formales, informales y no formales no es de oposición, es más bien de continuidad y complementariedad en la formación de los ciudadanos (Marandino, et al., 2008). Los autores señalan que los espacios de educación no formal se constituyen como instituciones educativas con proyectos más o menos estructurados y programas de estudios más flexibles. Desde la perspectiva del visitante y su formación, estas instituciones pueden tener características de la educación formal cuando la escuela complementa sus prácticas de enseñanza y aprendizaje con una actividad desarrollada en estos espacios, integrándolos en una secuencia didáctica (Ejemplo: Visita de una escuela a un zoológico para profundizar en el contenido de la biodiversidad). Y también puede tener características de la educación informal, cuando el visitante asiste a la institución con el propósito de ocio, integración familiar, conversaciones informales, etc.

\section{PROCESOS DE ENSEÑANZA Y APRENDIZAJE EN ZOOLÓGICOS}

Sobre la dimensión educativa en los zoológicos, The International Union of Directors of Zoological Gardens (1993) señala que uno de los objetivos que deben ser la base para la conservación de la biodiversidad está relacionada con la dimensión educativa de estos lugares. Los zoológicos deben promover un aumento de la conciencia pública, en relación con las políticas públicas de conservación, lo que determina el notable cambio de diseño de parques zoológicos como meramente destinados a mostrar animales hacia programas educativos relacionados con la educación ambiental y la conservación de la biodiversidad.

Gomes (2012) señala que hoy en día los zoológicos son instituciones enfocadas a la recreación y el ocio, pero esta práctica no es el único propósito de estos lugares, teniendo en cuenta la gran capacidad de éstos en las áreas de conservación, investigación y educación. El autor señala que parece que hay una falta de investigación sobre el uso de los zoológicos para enseñar la ciencia, aunque el potencial pedagógico de estas instituciones es reconocida por los educadores. 
Bio - grafía. Escritos sobre la Biología y su Enseñanza. ISSN 2027-1034

Edición Extraordinaria. p.p. 1469 - 1476

Memorias del IX Encuentro Nacional de Experiencias en Enseñanza de la Biología y la Educación Ambiental. IV Congreso Nacional de Investigación en Enseñanza de la Biología.

Particularmente, en el abordaje del concepto biodiversidad en espacios no formales de educación, una investigación realizada con 5561 visitantes de 26 zoológicos distribuidos en 19 países (Moss, Jensen, Gusset, 2014) reportó que después de visitar el Zoológico y participar de sus propuestas educativas, los visitantes incrementan su entendimiento sobre lo que es biodiversidad, así como sus conocimientos sobre acciones para contribuir en su protección.

\section{OBJETOS DE ESTUDIO Y POSIBILIDADES EN LA INVESTIGACIÓN DE LOS PROCESOS DE ENSEÑANZA EN ZOOLÓGICOS}

Transposición didáctica de conceptos biológicos y de la educación ambiental en Zoológicos: Salgados (2011) identificando lo que denominó de "transposición museográfica", se refiere al proceso de transposición y resignificación del conocimiento científico que ocurre en los espacios no formales de educación cuando se construye la exposición temática y cuando el mediador interactúa con los visitantes. Sobre la biodiversidad, el autor encontró que en los textos académicos la biodiversidad es descrita principalmente en el nivel de las especies, mientras que Acuarios y Zoológicos tienen una preocupación mayor por mostrar el comportamiento y hábitat de esas especies.

Adecuación de las instalaciones del Zoológico pensando en la experiencia del visitante: Genzini y de Almeida (2013) formulan que es importante cuestionarse, hasta qué punto las instalaciones de un espacio de educación no formal, invitan por si mismas a que el visitante explore libremente, indague, conecte ideas o se vincule emocional y cognitivamente. Pensar en estos aspectos podría permitir que se disminuya la distancia entre el significado que los elaboradores del espacio dan a la colección, y el significado que el visitante atribuye cuando la explora.

Evidencias de aprendizaje en Zoológicos: Es uno de los objetos de estudio más abordados en la investigación sobre enseñanza y aprendizaje en Zoológicos. Los autores recurren a comparar los conocimientos de los visitantes sobre algún asunto del Zoológico (por ejemplo, biodiversidad) antes y después de la visita. Kimble (2013) que los aprendizajes que los visitantes construyen, dependen directamente del tipo de actividad utilizada. Así mismo, encontró que los aprendizajes normalmente se relacionan con los nombres de las especies, la motivación, habilidades para la descripción, entre otros.

Proceso de aprendizaje, ¿Cómo se aprende en los Zoológicos? Queiróz y Fernandes (2015) han utilizado la perspectiva sociocultural para explorar las interacciones en diferentes niveles que acontecen en los espacios de educación no formal. Utilizan la estrategia de caracterizar las conversaciones que realizan los visitantes en la Institución, y clasificarlas como emotivas, conectivas, perceptivas y 
Bio - grafía. Escritos sobre la Biología y su Enseñanza. ISSN 2027-1034

Edición Extraordinaria. p.p. 1469 - 1476

Memorias del IX Encuentro Nacional de Experiencias en Enseñanza de la Biología y la Educación Ambiental. IV Congreso Nacional de Investigación en Enseñanza de la Biología.

conceptuales. De acuerdo con Angulo (2014) en los Museos, y en general, en los espacio de educación no formal, se debe dar la oportunidad para que los alumnos visitantes tengan la posibilidad de hablar, leer, escuchar y escribir sobre lo que están aprendiendo.

El mediador - Estrategias para enseñar y su formación inicial y continuada: Según García (2008) el conocimiento expresado en el discurso de los mediadores es el resultado de su conocimiento personal y también de sus experiencias vividas. Por ello, los espacios de educación no formal deben interesarse con la formación inicial y continuada de los mediadores. Considerando la interacción dialógica como un elemento clave para promover experiencias más significativas.

\section{PROPÓSTOS}

- Presentar aspectos generales de las diferencias y semejanzas entre los procesos educativos de espacios formales (escuelas, Universidades, Institutos) y espacios no formales de educación, principalmente Zoológicos.

- Discutir la relación Escuela-Zoológico para la enseñanza de la biología y la educación ambiental en el contexto colombiano a partir de la experiencia del Bioparque Wakatá, Parque Jaime Duque.

- Discutir posibilidades y desafíos en la investigación sobre los procesos de enseñanza y aprendizaje en Zoológicos y su contribución para la enseñanza de la Biología y la educación ambiental en espacios formales de educación.

- Estimular el uso de espacios no formales de educación para la enseñanza de las ciencias por parte de docentes y la investigación en el campo por parte de la comunidad académica de la didáctica.

\section{ASPECTOS PROCEDIMENT ALES}

\section{Público objetivo:}

- Profesores de ciencias naturales y biología en la educación primaria y secundaria.

- Docentes de biología y Ciencias Naturales en formación.

- Investigadores de la Didáctica de la biología y de las ciencias interesados en los procesos educativos en espacios no formales de educación.

- Integrantes de Departamentos de educación de espacios de educación no formal. 
Bio - grafía. Escritos sobre la Biología y su Enseñanza. ISSN 2027-1034

Edición Extraordinaria. p.p. 1469 - 1476

Memorias del IX Encuentro Nacional de Experiencias en Enseñanza de la Biología y la Educación Ambiental. IV Congreso Nacional de Investigación en Enseñanza de la Biología.

\section{Cantidad de personas:}

Un máximo de 25 personas.

\section{Duración:}

90-120 minutos

Detalle de las actividades

\begin{tabular}{|c|c|}
\hline Momento/Temática & Descripción de la actividad \\
\hline $\begin{array}{l}\text { Momento } 1 \\
\text { Educación formal y no formal: } \\
\text { ¿Relación de oposición o } \\
\text { continuidad? } \\
20 \text { minutos }\end{array}$ & $\begin{array}{l}\text { Presentación de los conceptos de educación } \\
\text { formal y no formal, diferencias y semejanzas en } \\
\text { los siguientes aspectos: Propuestas } \\
\text { curriculares, evaluación, tiempo de enseñanza, } \\
\text { actores que participan de los procesos. }\end{array}$ \\
\hline $\begin{array}{l}\text { Momento } 2 \\
\text { Estrategias para promover la } \\
\text { aproximación entre escuelas y } \\
\text { Zoológicos, identificando } \\
\text { propósitos comunes. } \\
35 \text { minutos }\end{array}$ & $\begin{array}{l}\text { A través de una rueda de conversación, los } \\
\text { participantes dialogan sobre dos aspectos: } \\
\text { a) Los principales desafíos y principales } \\
\text { problemáticas de la enseñanza de la Biología y } \\
\text { de la Educación Ambiental en el contexto actual } \\
\text { del país. } \\
\text { b) Las expectativas que tienen sobre los } \\
\text { Zoológicos y el potencial pedagógico de los } \\
\text { espacios no formales de educación. } \\
\text { A partir del dialogo establecido entre los } \\
\text { participantes, se discutirá sobre las } \\
\text { posibilidades y desafíos de la integración entre } \\
\text { espacios de educación formal y no formal para } \\
\text { el abordaje de las dificultades de la enseñanza } \\
\text { de la Biología y la Educación Ambiental en el } \\
\text { contexto actual del país. }\end{array}$ \\
\hline $\begin{array}{c}\text { Momento } 3 \\
\text { Enseñanza de la Biología y la } \\
\text { Educación Ambiental en } \\
\text { Espacios no Formales de } \\
\text { educación, un campo de } \\
\text { investigación por explorar en } \\
\text { Colombia. } \\
50 \text { minutos }\end{array}$ & $\begin{array}{l}\text { Presentación del Departamento de educación } \\
\text { del Bioparque Wakatá (Parque Jaime Duque) y } \\
\text { sus actividades de integración con escuelas del } \\
\text { Departamento de Cundinamarca. } \\
\text { Discusión de algunos trabajos desarrollados en } \\
\text { el área de la investigación sobre los procesos } \\
\text { de enseñanza y aprendizaje en los espacios de } \\
\text { educación no formal en el contexto colombiano. }\end{array}$ \\
\hline
\end{tabular}


Bio - grafía. Escritos sobre la Biología y su Enseñanza. ISSN 2027-1034

Edición Extraordinaria. p.p. 1469 - 1476

Memorias del IX Encuentro Nacional de Experiencias en Enseñanza de la Biología y la Educación Ambiental. IV Congreso Nacional de Investigación en Enseñanza de la Biología.

\begin{tabular}{|c|c|}
\hline & $\begin{array}{c}\text { Presentación de líneas de trabajo que pueden } \\
\text { ser abordadas en la investigación en esta área. }\end{array}$ \\
\hline Discusión final. & \\
Cierre. & \\
15 Minutos & \\
\hline
\end{tabular}

\section{Materiales y recursos}

- Sala para 25 personas.

- 25 Sillas

- Equipos audiovisuales (videobeam, o televisor con audio)

- 50 hojas adhesivas tipo post it.

- 25 Esferos o lápices.

\section{Bibliografía}

Angulo, F. (2014). El papel mediador del museo de ciencias en la estructuración y síntesis del aprendizaje. Bio-grafía. Escritos sobre la Biología y su enseñanza, v. 8, n.15, p. $123-128$.

Bianconi, M.; Caruso, F. (2005) Apresentação: Educação não formal. Ciência e cultura. V. 57, n. 4.

Cazelli, S. (2005). Ciência, Cultura, Museus, Jovens e Escolas: quais as relações? 2005. Tese (Doutorado em Educação) - Pontifícia Universidade Católica do Rio de Janeiro, Rio de Janeiro.

Fachín, A.; Moreira, R.; Balieiro, H.; Santos, A. \& García, A. (2011) A caracterização dos espaços não formais de educação científica para o ensino de ciências. Revista Amazônica de Ensino de Ciências. V. 4, n. 4, p. 12-23.

Garcia, V. A. R. (2008) O processo de aprendizagem no zoológico de Sorocaba: análise da atividade educativa visita orientada a partir dos objetos biológicos. Dissertação (Mestrado em Educação) Faculdade de Educação da Universidade de São Paulo, São Paulo.

Genzini, T., \& De Almeida, J. (2013). Comunicação pedagógica em museus de ciências. In: Atas do IX Encontro Nacional de Pesquisa em Educação em Ciências - IX ENPEC, Águas de Lindóia.

Gomes, A. (2012) O Jardim Zoológico enquanto espaço não formal para promoção do desenvolvimento de etapas do raciocínio científico. Dissertação, Universidade de Brasilia. Brasilia.

Kimble, J. (2013). Children learning about biodiversity at an environment Centre, a museum and at live animal shows. Studies in educational evaluation. 
Bio - grafía. Escritos sobre la Biología y su Enseñanza. ISSN 2027-1034

Edición Extraordinaria. p.p. 1469 - 1476

Memorias del IX Encuentro Nacional de Experiencias en Enseñanza de la Biología y la Educación Ambiental. IV Congreso Nacional de Investigación en Enseñanza de la Biología.

International Union Of Directors Of Zoological Gardens. (1993) The world zoo conservation strategy: the role of the zoo and aquaria of the world in global conservation. Illinois: Chicago Zoological Society.

Marandino, M. (Org.); Fernandes, A.; Navas, A.; Contier, D.; Standerski, L.; Magalhaes, L.; Conrado, L.; Correia, M. E Rachid, V. (2008) Educação em Museus: A mediação em foco. São Paulo: FEUSP.

Moss, A., Jensen, E., \& Gusset, M. (2014). Evaluating the Contribution of Zoos and Aquariums to Aichi Biodiversity Target 1. Conservation biology, 0(0), 1-8.

Queiróz, H., \& Fernandes, A. (2015). "Conversas de aprendizagem" em zoológicos e suas relações com a conservação da Biodiversidade. In: Atas do X Encontro Nacional de Pesquisa em Educação em Ciências - X ENPEC, Águas de Lindóia. 